\title{
Structural Models for Flight Dynamic Analysis of Very Flexible Aircraft
}

\author{
Rafael Palacios ${ }^{1}$ \\ Imperial College, London SW7 2AZ, UK \\ Carlos E.S. Cesnik ${ }^{2}$ \\ University of Michigan, Ann Arbor, MI 48109, USA
}

\begin{abstract}
Dissimilar analysis models are considered for the large structural deformations of aircraft with high-aspect-ratio composite wings. The different approaches include displacementbased, strain-based, and intrinsic geometrically-nonlinear beam models. Comparisons are made in terms of numerical efficiency and simplicity for integration of full aircraft flexibility in flight dynamics models. An analysis procedure is proposed based on model substructuring with a (linear) modal representation of both fuselage and tail and (nonlinear) intrinsic beam elements for the flexible wings.
\end{abstract}

\section{Introduction}

$\mathrm{T}$ HIS paper addresses the problem of computational efficiency in the modeling of the geometrically-nonlinear structural deformations involved in flight dynamics of very flexible aircraft (VFA). This is done in the context of the newly proposed ultra-long-endurance Unmanned Aerial Vehicles (UAVs), which typically present composite wings of very high aspect ratio. High flexibility becomes then a major concern with several major implications in any engineering analysis: First, it should be able to handle large deformations of those very flexible primary structures; second, it should consider flow characteristics determined by large motions of the wetted surfaces relative to mean flow speed, including the likely possibility of wing stall; and third, vehicle vibrations will occur at very low frequencies, which will easily get coupled with the time scales of the flight dynamics response. As all three problems are intrinsically nonlinear, performance prediction for long-endurance UAVs becomes a coupled problem involving the vehicle nonlinear structural, aero-, and flight dynamics.

The development of large-scale very lightweight UAVs has therefore brought a renewed interest into the flight dynamics of flexible aircraft. This needs to expand the quasi-static approach used in the evaluation of the basic aerodynamic derivatives of large aircraft ${ }^{1}$, although in most cases the work has focused on the rigid body equations augmented with linear structural models ${ }^{2-3}$. While this approach is valid in a majority of situations, it is not sufficient

\footnotetext{
${ }^{1}$ Lecturer, Department of Aeronautics, 355 Roderic Hill Building (rpalacio@imperial.ac.uk). Member AIAA

${ }^{2}$ Professor, Department of Aerospace Engineering, 3024 FXB Building (cesnik@umich.edu). Associate Fellow, AIAA
} 
for a complete analysis of VFA with large changes on the inertia properties, as illustrated by the mishap of the Helios $^{4}$.

The pioneer work to include geometrically-nonlinear effects in the deformation of low-stiffness aircraft was conducted in the development of the Daedalus human-powered aircraft ${ }^{5}$. This aircraft had very restricted flight conditions and nonlinear effects were only included in the static aeroelastic analysis, while the dynamic response of the aircraft was based on linear models. Still, the large deformations were shown to have a large impact in the actual flight dynamics characteristics of the aircraft. Long-endurance aircraft will have to operate in less restricted flight conditions than the Daedalus. For their design to be practical, aircraft need to be designed to withstand certain levels of atmospheric turbulence, which for a VFA means that a geometrically-nonlinear structural dynamics model is needed. Different groups $^{6-8}$ have been recently developing such models based on the coupling of geometricallynonlinear beams and 2-D unsteady airfoil aerodynamics for the high-aspect-ratio wings, and the flight dynamics of the full vehicle. This allows capturing the basic physical phenomena with a relatively small size of the problem and has provided significant evidence on the large effect that structural deformations may have on the aircraft dynamics characteristics (typically, the short period and phugoid modes).

Ref. 9 presents a review of the most important aspects in which the response of VFA is affected by a nonlinear structural behavior. It includes the longitudinal stability characteristics, body-freedom flutter, response to gust and turbulence loads, and definition of control laws. Based on that, Ref. 9 reviewed the requirements in the definition of numerical models that would be needed to appropriately describe the dynamics of very flexible aircraft. It emphasizes the importance of computationally-efficient solution procedures with a limited number of degrees of freedom, in order to account for the interactions between structural, aero- and flight dynamics in long-term dynamic simulations (for instance, in analysis of complex maneuvers or optimal path tracking). Strain-based beam models ${ }^{10}$ are particularly suitable for this type of application: For static problems, they simply need the inversion of a constant matrix, and for dynamic problems with low-frequency content they will have very fast convergence rates. For them to be of practical use, they need to be accompanied by a high-fidelity dimensional reduction procedure ${ }^{11}$ from the actual 3-D solid mechanics problem. In the aerodynamics, time-domain unsteady models ${ }^{12}$ need to include dynamic stall effects to address the likely occurrence of localized stall in the large excursions of the wing sections ${ }^{13}$. Finally, the time integration of the coupled equations needs to be performed by an efficient yet accurate integration scheme for the nonlinear system of equations ${ }^{14}$.

In this paper we investigate different alternatives for the geometrically-nonlinear structural model targeted for multidisciplinary analysis of VFA. For that purpose, different beam models are considered that accounts for the large structural deformations. Their numerical efficiency in the context of a full aircraft analysis is studied, including reduced-order approaches. An approach is finally proposed based on model substructuring with a modal representation of both fuselage and tail and an intrinsic formulation for the wings. 


\section{Basic structural models}

The geometrically-nonlinear dynamic equations of motion of a curved beam of arc-length $l$ in the time interval $\left[t_{1}, t_{2}\right]$ can be written, in weak form, as ${ }^{16}$

$$
\int_{t_{1}}^{t_{2}} \int_{0}^{l}\left[\delta V_{B}^{T} P_{B}+\delta \Omega_{B}^{T} H_{B}-\delta \gamma^{T} F_{B}-\delta \kappa^{T} M_{B}+\delta R_{a}^{T} f_{a}+\delta \Phi_{B}^{T} m_{B}\right] d s d t=0
$$

Sub-indexes are used to indicate the coordinate system in which each vector magnitude is projected. $a$ is a (moving) common reference frame with prescribed linear and angular velocities $v_{a}$ and $\omega_{a}$, respectively, while $B$ is the local co-ordinate system along the deformed beam reference line. We follow the nomenclature laid out in Ref. 16. The relations between internal forces/moments $\left(F_{B}, M_{B}\right)$ and corresponding strains/curvatures $(\gamma, \kappa)$, as well as between linear and angular momenta $\left(P_{B}, H_{B}\right)$ and corresponding linear and angular velocities $\left(V_{B}, \Omega_{B}\right)$, are assumed to be given by linear constitutive relations,

$$
\begin{aligned}
& P_{B}=\mathrm{m} V_{B}-\mathrm{m} \tilde{\xi}_{c g} \Omega_{B}, \\
& H_{B}=\mathrm{m} \tilde{\xi}_{c g} V_{B}+J \Omega_{B},
\end{aligned} \quad \text { and } \quad \begin{aligned}
& \gamma=c_{\gamma f} F_{B}+c_{\gamma m} M_{B}, \\
& \kappa=c_{\kappa f} F_{B}+c_{\kappa m} M_{B} .
\end{aligned}
$$

These equations correspond to an arbitrary location of the beam reference line and anisotropic (composite) material distributions ${ }^{11}$. They need to be solved together with the velocity-displacement and the strain-displacement kinematic relations, which for a geometrically-exact beam are, respectively ${ }^{16}$,

$$
\begin{aligned}
& V_{B}=C^{B a}\left(\dot{R}_{a}+\tilde{\omega}_{a} R_{a}+v_{a}\right), \quad \text { and } \quad \gamma=C^{B a} R_{a}^{\prime}-e_{1} \text {, } \\
& \tilde{\Omega}_{B}=C^{B a} \dot{C}^{a B}+C^{B a} \tilde{\omega}_{a} C^{a B}, \quad \text { and } \quad \tilde{\kappa}=C^{B a}\left(C^{a B}\right)^{\prime}-\tilde{k}_{b} \text {, }
\end{aligned}
$$

where $R_{a}$ is the position vector of a point along the reference line, $C^{B a}$ is the rotation matrix from the reference to the local frame, $k_{b}$ is the initial curvature of the local undeformed frame (denoted by $b$ ) and $e_{i}$ are the unit vectors. Dots $(\bullet)$ denote derivatives with time, and primes $\left(\bullet^{\prime}\right)$ derivatives with the curvilinear coordinate, $s$. Eqs. (1)-(3) are complemented by the corresponding initial and boundary conditions. Several solution procedures can be devised to solve the problem. We will consider here three of them, all based on the finite-element method, but with each one using a different selection of independent degrees of freedom, that is, displacements, strain, and velocities/strains. We will call them $d$-, $s$ - and $i$-beam elements, respectively.

\section{A. Displacement-based beam element ( $d$-beams)}

Displacements and rotations of the reference line constitute in this case the irreducible set of independent variables. Eq. (1) can be written in strong form as ${ }^{16}$

$$
\begin{aligned}
& \left(\frac{d}{d t}+\tilde{\Omega}_{B}\right) P_{B}=\left(\frac{d}{d x}+\tilde{K}_{B}\right) F_{B}+f_{B}, \\
& \left(\frac{d}{d t}+\tilde{\Omega}_{B}\right) H_{B}+\tilde{V}_{B} P_{B}=\left(\frac{d}{d x}+\tilde{K}_{B}\right) M_{B}+\left(\tilde{e}_{1}+\tilde{\gamma}\right) F_{B}+m_{B},
\end{aligned}
$$


which are solved together with the appropriate set of boundary conditions. A parameterization of the finite rotation is now needed (Euler or Bryant angles, Rodrigues parameters, quaternions, etc.). We have chosen, as in Ref. 15, the Cartesian rotation vector, $\Psi$, in the rotation from coordinate system $a$ to the local $B$ frame. Next, we substitute Eq. (2) into (3) and then into the equilibrium equations (4) to define a closed set of equations on the state variable

$$
\eta=\left\{\begin{array}{l}
R_{a} \\
\Psi
\end{array}\right\}
$$

which is finally approximated by a finite-element discretization, i.e., $\eta=N \bar{\eta}$, where $\bar{\eta}$ is the discrete set of degrees of freedom and $N$ the corresponding shape functions. In particular, a 3-noded curved beam element has been implemented. With these definitions, Eq. (4) can be written as ${ }^{15}$

$$
F=\mathcal{M}_{\eta}(\bar{\eta}) \ddot{\bar{\eta}}+F_{g y r}\left(\bar{\eta}, \dot{\bar{\eta}}, v_{a}, \dot{v}_{a}, \omega_{a}, \dot{\omega}_{a}\right)+F_{s t i f}(\bar{\eta})-F_{e x t}=0
$$

where $\mathcal{M}_{\eta}$ is the discrete mass matrix and $F_{g y r}, F_{s t i f}$ and $F_{e x t}$ are the discrete gyroscopic, stiffness, and external generalized forces, respectively. This can be considered as the conventional approach to create a geometricallynonlinear beam element, what we will call the $d$-beam element, and for which various implementations can be found in the literature ${ }^{15,20}$. It has several advantages: it directly provides the solution in the physical degrees of freedom in the problem for aeroelastic or flexible-body dynamic problems, its linearized version yields the usual symmetric matrices, and a number of efficient solution algorithms (Newmark, HHT, Generalized- $\alpha$ ), are readily available. However, solving directly the rotational degree of freedom is a costly numerical exercise. The number of operations to build the different functionals in Eq. (6) is relatively high and convergence of implicit algorithms based on Newton-Raphson procedures is relatively slow. In addition to this, the mass matrix and the different functionals need to be regularly updated in the solution.

\section{B. Strain-based beam element (s-beams)}

An alternative solution path is obtained by taking the strains defined in Eq. (3) as the independent variables in the problem and define them to be piecewise constant in the discretization of the reference line ${ }^{10}$. For that purpose, Eq. (3) is first rewritten as

$$
\begin{gathered}
R_{a}^{\prime}=C^{a B}\left(e_{1}+\gamma\right), \\
C^{a B^{\prime}}=C^{a B} \tilde{K}_{B} .
\end{gathered}
$$

If $\gamma=\gamma_{n}$ and $\kappa=\kappa_{n}$ are constant along the segment $s_{n-1} \leq s<s_{n}$, Eq. (7) can be directly integrated as

$$
\begin{gathered}
R_{a}(s)=R_{a}\left(s_{n-1}\right)+C^{a B}\left(s_{n-1}\right) H^{1}\left(\kappa_{n}, s-s_{n-1}\right)\left(e_{1}+\gamma_{n}\right), \\
C^{a B}(s)=C^{a B}\left(s_{n-1}\right) H^{0}\left(\kappa_{n}, s-s_{n-1}\right),
\end{gathered}
$$

with 


$$
\begin{aligned}
& H^{0}(\kappa, \Delta s)=e^{\widetilde{\Delta \Psi}}=I+\frac{\sin (\Delta \phi)}{\Delta \phi} \widetilde{\Delta \Psi}+\frac{1-\cos (\Delta \phi)}{\Delta \phi^{2}} \widetilde{\Delta \Psi} \widetilde{\Delta \Psi}, \\
& H^{1}(\kappa, \Delta s)=\Delta s\left(I+\frac{1-\cos (\Delta \phi)}{\Delta \phi^{2}} \widetilde{\Delta \Psi}+\frac{\Delta \phi-\sin (\Delta \phi)}{\Delta \phi^{3}} \widetilde{\Delta \Psi} \widetilde{\Delta \Psi}\right)
\end{aligned}
$$

where $\Delta \Psi=K_{B} \Delta s=\left(k_{b}+\kappa\right) \Delta s$ and $\Delta \phi=\|\Delta \Psi\|$. For small values of $\Delta \phi$, these functions can be approximated as

$$
\begin{aligned}
& H^{0}(\kappa, \Delta s)=I+\widetilde{\Delta \Psi}+\frac{1}{2} \widetilde{\Delta \Psi} \widetilde{\Delta \Psi}+o\left(\Delta \phi^{2}\right) \\
& H^{1}(\kappa, \Delta s)=\Delta s I+\frac{\Delta s}{2} \widetilde{\Delta \Psi}+\frac{\Delta s}{6} \widetilde{\Delta \Psi} \widetilde{\Delta \Psi}+o\left(\Delta s \Delta \phi^{2}\right)
\end{aligned}
$$

Note that there is no need to assume that shear strains are zero, i.e., $\gamma_{12}=0, \gamma_{13}=0$, as it was done in Ref. 10 and subsequent works ${ }^{8}$, although in most practical cases that approximation is in fact justified. These expressions allow the discretization of the reference line using constant-strain elements of length $\Delta s_{n}=s_{n}-s_{n-1}$. We define now

$$
\begin{gathered}
H_{n}^{x}=H^{x}\left(\kappa_{n}, \Delta s_{n}\right), \\
H_{s}^{x}=H^{x}\left(\kappa_{n}, s-s_{n-1}\right) .
\end{gathered}
$$

The position vector and orientation within the element $n$ (i.e., $s_{n-1} \leq s<s_{n}$ ) can be then obtained as

$$
\begin{gathered}
R_{a}(s)=R_{a 0}+C_{0}^{a B} H_{1}^{1}\left(e_{1}+\gamma_{1}\right)+C_{0}^{a B} \sum_{e=2}^{n-1} H_{1}^{0} \cdots H_{e-1}^{0} H_{e}^{1}\left(e_{1}+\gamma_{e}\right)+C_{0}^{a B} H_{1}^{0} \cdots H_{n-1}^{0} H_{s}^{1}\left(e_{1}+\gamma_{n}\right), \\
C^{a B}(s)=C_{0}^{a B} H_{1}^{0} \cdots H_{n-1}^{0} H_{s}^{0} .
\end{gathered}
$$

For a given value of the position vector, $R_{a 0}$, and orientation, $C_{0}^{a B}$, at $s=s_{0}$ (i.e., the boundary values), Eqs. (12) provide a closed-form expression for the position vector and orientation as function of the beam strain distribution. Discontinuities in curvature of the discrete reference line can be easily included as additional (constant) rotations between the elements that are linked to a given node. Eqs. (12) are valid only along open kinematic chains (also known as tree in the rigid-body dynamics literature). For closed chains, these equations need to be complemented by the appropriate constraints, which can be defined by Lagrange multipliers ${ }^{17}$, but this is not relevant in many aircraft applications and will not be discussed here. Differentiation in the previous equations gives the virtual displacements and rotations as function of the virtual strains, that is,

$$
\begin{gathered}
\delta R_{a}(s)=\delta R_{a 0}+\delta C_{0}^{a B} C_{0}^{B a}\left(R_{a}(s)-R_{a 0}\right)+C_{0}^{a B}\left[H_{1}^{1} \delta \gamma_{1}+\sum_{e=2}^{n-1} H_{1}^{0} \cdots H_{e}^{0} \delta \gamma_{e}+H_{1}^{0} \cdots H_{n-1}^{0} H_{s}^{1} \delta \gamma_{n}\right]+ \\
+C_{0}^{a B}\left[\partial_{\kappa_{1}} H_{1}^{1} \delta \kappa_{1}\left(e_{1}+\gamma_{1}\right)+\sum_{e=2}^{n-1} H_{1}^{0} \cdots \partial_{\kappa_{e}} H_{e}^{0} \delta \kappa_{e} \cdots H_{s}^{1}\left(e_{1}+\gamma_{e}\right)+H_{1}^{0} \cdots H_{n-1}^{0} \partial_{\kappa_{n}} H_{s}^{1} \delta \kappa_{n}\left(e_{1}+\gamma_{n}\right)\right] \\
\delta C^{a B}(s)=\delta C_{0}^{a B} C_{0}^{B a} C^{a B}(s)+ \\
+C_{0}^{a B} \sum_{e=1}^{n-1} H_{1}^{0} \cdots \partial_{\kappa_{e}} H_{e}^{0} \delta \kappa_{e} \cdots H_{s}^{0}+C_{0}^{a B} H_{1}^{0} \cdots H_{n-1}^{0} \partial_{\kappa_{n}} H_{s}^{0} \delta \kappa_{n} .
\end{gathered}
$$

In compact form, these expressions can be written, after some manipulation, as 


$$
\begin{gathered}
\delta R_{a}(s)=\delta R_{a 0}+\delta C_{0}^{a B} C_{0}^{B a}\left(R_{a}(s)-R_{a 0}\right)+C_{0}^{a B}\left(\mathcal{H}_{\gamma}^{1}(\bar{\kappa}, s) \delta \bar{\gamma}+\mathcal{H}_{\kappa}^{1}(\bar{\gamma}, \bar{\kappa}, s) \delta \bar{\kappa}\right), \\
\delta C^{a B}=\delta C_{0}^{a B} C_{0}^{B a} C^{a B}(s)+C_{0}^{a B} \mathcal{H}_{\kappa}^{0}(\bar{\kappa}, s) \delta \bar{\kappa}
\end{gathered}
$$

where we have introduced the following vectors of the independent degrees of freedom in the discretization of the problem

$$
\bar{\gamma}=\left\{\begin{array}{c}
\gamma_{1} \\
\vdots \\
\gamma_{N}
\end{array}\right\}, \quad \bar{\kappa}=\left\{\begin{array}{c}
\kappa_{1} \\
\vdots \\
\kappa_{N}
\end{array}\right\}
$$

Note that $\mathcal{H}_{\kappa}^{0}$ in Eq. (15) is a third-order tensor. Similar expressions to Eq. (15) are obtained for the time derivatives of the position vector and rotation matrix, as

$$
\begin{aligned}
& \dot{R}_{a}=\dot{R}_{a 0}+\dot{C}_{0}^{a B} C_{0}^{B a}\left(R_{a}-R_{a 0}\right)+C_{0}^{a B}\left(\mathcal{H}_{\gamma}^{1} \dot{\bar{\gamma}}+\mathcal{H}_{\kappa}^{1} \dot{\bar{\kappa}}\right), \\
& \dot{C}^{a B}=\dot{C}_{0}^{a B} C_{0}^{B a} C^{a B}+C_{0}^{a B} \mathcal{H}_{\kappa}^{0} \dot{\bar{\kappa}} .
\end{aligned}
$$

We are finally ready to write the equations of motion for the problem. For simplicity, we will assume in what follows that the beam reference line is located at the center of mass of each cross section $\left(\xi_{c g}=0\right)$. Under that assumption and for prescribed motion of the reference frame, $a$, Eq. (1) is written as

$$
\begin{aligned}
\int_{t_{1}}^{t_{2}} \int_{0}^{l}\left\{\delta R_{a}^{T}\left(\mathrm{~m} \dot{V}_{a}+\mathrm{m} \tilde{\omega}_{a} V_{a}-f_{a}\right)+\delta \gamma^{T} F_{B}+\delta \kappa^{T} M_{B}+\right. \\
\left.\quad-\frac{1}{2} \operatorname{tr}\left[\delta C^{B a} C^{a B}\left(\widetilde{J \dot{\Omega}_{B}}+\overline{J \tilde{\Omega}_{B} \Omega_{B}}-\tilde{m}_{B}\right)\right]\right\} d s d t=-\int_{0}^{l}\left[\mathrm{~m} \delta R_{a}^{T} V_{a}+\delta \Phi_{B}^{T} J \Omega_{B}\right]_{t_{1}}^{t_{2}} d s,
\end{aligned}
$$

where we have used the relation $\alpha^{T} \beta=-\frac{1}{2} \operatorname{tr}(\tilde{\alpha} \tilde{\beta})$. If we further assume that the reference frame for the beam motion is the beam local frame at $s=0$, that is, $a \equiv B_{0}$, then it is $R_{a 0}=0$ and $C_{0}^{B a}=I$. Eq. (17) then simplifies to

$$
\begin{aligned}
& \dot{R}_{a}=\mathcal{H}_{\gamma}^{1} \dot{\bar{\gamma}}+\mathcal{H}_{\kappa}^{1} \dot{\bar{\kappa}}, \\
& \ddot{R}_{a}=\mathcal{H}_{\gamma}^{1} \ddot{\bar{\gamma}}+\mathcal{H}_{\kappa}^{1} \ddot{\bar{\kappa}}+\dot{\mathcal{H}}_{\gamma}^{1} \dot{\bar{\gamma}}+\dot{\mathcal{H}}_{\kappa}^{1} \dot{\bar{\kappa}}, \\
& \dot{C}^{a B}=\mathcal{H}_{\kappa}^{0} \dot{\bar{\kappa}}, \\
& \ddot{C}^{a B}=\mathcal{H}_{\kappa}^{0} \ddot{\bar{\kappa}}+\dot{\mathcal{H}}_{\kappa}^{0} \dot{\bar{\kappa}} .
\end{aligned}
$$

Thus the relative velocities of the beam elements with respect to a reference frame at $s=0$ are only a function of the internal strain along the beam reference line. Substituting Eq. (19) into the first of Eqs. (3) and their time derivatives, we finally obtain the dynamic equations that provide the beam strains as a function of the boundary velocities and the applied forces

$$
\mathcal{M}_{s}(\bar{\gamma}, \bar{\kappa})\left\{\begin{array}{c}
\ddot{\bar{\gamma}} \\
\ddot{\bar{\kappa}}
\end{array}\right\}+C_{s}(\bar{\gamma}, \bar{\kappa})\left\{\begin{array}{c}
\dot{\bar{\gamma}} \\
\overline{\bar{\kappa}}
\end{array}\right\}+\mathcal{K}_{s}\left\{\begin{array}{c}
\bar{\gamma} \\
\bar{\kappa}
\end{array}\right\}+\mathcal{M}_{g y r}(\bar{\gamma}, \bar{\kappa})\left\{\begin{array}{c}
\dot{v}_{a} \\
\dot{\omega}_{a}
\end{array}\right\}+C_{g y r}\left(\bar{\gamma}, \bar{\kappa}, \dot{\bar{\gamma}}, \dot{\bar{\kappa}}, \omega_{a}\right)\left\{\begin{array}{c}
v_{a} \\
\omega_{a}
\end{array}\right\}=\left\{\begin{array}{c}
f \\
m
\end{array}\right\} .
$$


Note that the static part of Eq, (20) defines a linear system and still accounts for large displacements and rotations. This is relevant in very flexible aircraft analysis, as the large contribution to the deformation comes from the aircraft trim loads. The update of the matrices for the dynamics analysis can be done in an adaptive manner ${ }^{14}$.

\section{Intrinsic beam element (i-beams)}

Finally, a hybrid (or mixed) solution procedure can be also defined. Ref. 16 introduced a three-field solution, in which Eqs. (1), (2) and (3) are simultaneously enforced using Lagrange multipliers. This simplifies the solution process, as compared with the displacement-based solution of Eq. (4), by requiring simpler interpolation functions, but increases the number of states in the problem. Moreover, retaining the rotation degrees of freedom as independent degrees is in general a costly option. A more interesting approach is a two-field solution ${ }^{19}$ based on strains (or internal forces) and velocities along the reference line. In this case, Eq. (4) is complemented by an equation that results from eliminating the position vector and the rotation matrix in Eq. (3). This is nothing more than a compatibility equation that imposes that the successive derivatives of the displacement/rotation field in time and space have can be defined in any order. It results in the following system of equations ${ }^{19}$,

$$
\begin{gathered}
\dot{P}+\tilde{\Omega} P=F^{\prime}+\tilde{K} F+f, \\
\dot{H}+\tilde{\Omega} H+\tilde{V} P=M^{\prime}+\tilde{K} M+\left(\tilde{e}_{1}+\tilde{\gamma}\right) F+m, \\
\dot{\gamma}=V^{\prime}+\tilde{K} V+\left(\tilde{e}_{1}+\tilde{\gamma}\right) \Omega \\
\dot{\kappa}=\Omega^{\prime}+\tilde{K} \Omega .
\end{gathered}
$$

It is assumed that all vector components are projected into the local deformed frame $(B)$ and therefore the subindex has been dropped. Eqs. (21), together with the constitutive relations (2), provide a closed-form solution to the problem. Displacements and rotations are dependent variables which can be obtained at each converged iteration by Eq. (8). Note that this formulation only involves first-order differential equations. The major advantage of this approach with respect to the previous one is that it reduces both the number of operations per iteration and the bandwidth of the matrices in the solution, which results therefore in significantly less expensive problem to update for full aircraft analyses. Note also that the reference system rigid-body velocities, $v_{a}$ and $\omega_{a}$, and their derivatives no longer explicitly appear in the equilibrium equation. In weak form, and after substituting the constitutive relations (2), Eq. (21) can be written as

$$
\begin{gathered}
\int_{t_{1}}^{t_{2}} \int_{0}^{l}\left\{\delta R^{T}\left[\mathrm{~m} \dot{V}-\mathrm{m} \tilde{\xi}_{c g} \dot{\Omega}+\mathrm{m} \tilde{\Omega}\left(V-\tilde{\xi}_{c g} \Omega\right)-F^{\prime}-\tilde{K} F-f\right]+\right. \\
+\delta \Phi^{T}\left[\mathrm{~m} \tilde{\xi}_{c g} \dot{V}+J \dot{\Omega}+\mathrm{m} \tilde{\xi}_{c g} \tilde{\Omega} V+\tilde{\Omega} J \Omega-M^{\prime}-\tilde{K} M-\left(\tilde{e}_{1}+\tilde{\gamma}\right) F-m\right]+ \\
+\delta I^{T}\left[c_{\gamma f} \dot{F}+c_{\gamma m} \dot{M}-V^{\prime}-\tilde{K} V-\left(\tilde{e}_{1}+\tilde{\gamma}\right) \Omega\right]+ \\
\left.+\delta J^{T}\left[c_{\kappa f} \dot{F}+c_{\kappa m} \dot{M}-\Omega^{\prime}-\tilde{K} \Omega\right]\right\} d s d t=0
\end{gathered}
$$

where we have introduced the infinitesimal rotation $\widetilde{\delta \Phi}=C^{B a} \delta C^{a B}$, as well as the force and moment impulse variations, $\delta I$ and $\delta \mathcal{J}$, respectively. They will also have to satisfy the boundary conditions. For instance, for a beam clamped at $s=0$ and free at $s=l$, it will be $\delta R(0)=0, \delta \Phi(0)=0, V(0)=0, \Omega(0)=0$, and $\delta I(l)=0$, 
$\delta \mathcal{J}(l)=0, F(l)=0, M(l)=0$, respectively. A finite-element approximation to solve Eq. (22) is introduced now on both the state variables and the Lagrange multipliers (infinitesimal variations) as

$$
\begin{gathered}
\left\{\begin{array}{l}
V \\
\Omega
\end{array}\right\}=N\left\{\begin{array}{l}
\bar{V} \\
\bar{\Omega}
\end{array}\right\}, \quad\left\{\begin{array}{l}
F \\
M
\end{array}\right\}=N\left\{\begin{array}{l}
\bar{F} \\
\bar{M}
\end{array}\right\}, \\
\left\{\begin{array}{l}
\delta R \\
\delta \Phi
\end{array}\right\}=W\left\{\begin{array}{l}
\delta \bar{R} \\
\delta \bar{\Phi}
\end{array}\right\}, \quad\left\{\begin{array}{l}
\delta I \\
\delta \mathcal{J}
\end{array}\right\}=W\left\{\begin{array}{l}
\delta \bar{I} \\
\delta \overline{\mathcal{J}}
\end{array}\right\} .
\end{gathered}
$$

In the assembly process, a master-slave approach has been used here for elements which share a node: One of the elements was taken as reference and defines the degrees of freedom for a particular node in the global arrays, while the other elements become slaves on that shared node with its vector magnitudes defined by a constant rotation from the master element. We will assume that the relative rotations between element variables at each node have been already embedded in Eq. (23). With these definitions, Eq. (22) can be rewritten as

$$
\begin{gathered}
\mathcal{M} \frac{d}{d t}\left\{\begin{array}{l}
\bar{V} \\
\bar{\Omega}
\end{array}\right\}+Q_{g y r}(\bar{V}, \bar{\Omega})+Q_{s t i f}(\bar{F}, \bar{M})=Q_{e x t}, \\
\mathcal{T}_{1} \frac{d}{d t}\left\{\begin{array}{l}
\bar{F} \\
\bar{M}
\end{array}\right\}+\mathcal{T}_{\gamma}(\bar{F}, \bar{M})\left\{\begin{array}{l}
\bar{V} \\
\bar{\Omega}
\end{array}\right\}=0 .
\end{gathered}
$$

The first one is the equation of motion, while the second is the kinematic compatibility equation. The matrices in this last equation are

$$
\mathcal{T}_{1}=\int_{0}^{l} W^{T}\left[\begin{array}{cc}
c_{\gamma f} & c_{\gamma m} \\
c_{\kappa f} & c_{\kappa m}
\end{array}\right] N d s, \quad \mathcal{T}_{\gamma}=-\int_{0}^{l} W^{T} N^{\prime} d s-\int_{0}^{l} W^{T}\left[\begin{array}{cc}
\tilde{K} & \tilde{e}_{1}+\tilde{\gamma} \\
0 & \tilde{K}
\end{array}\right] N d s .
$$

The mass matrix in the equation of motion is a constant matrix, defined as

$$
\mathcal{M}=\int_{0}^{l} W^{T}\left[\begin{array}{cc}
\mathrm{m} & -\mathrm{m} \tilde{\xi}_{c g} \\
\mathrm{~m} \tilde{\xi}_{c g} & J
\end{array}\right] N d s .
$$

The discrete gyroscopic, stiffness, and applied forces in the equation of motion are, respectively,

$$
Q_{g y r}=\int_{0}^{l} W^{T}\left\{\begin{array}{c}
\mathrm{m} \tilde{\Omega}\left(V-\tilde{\xi}_{c g} \Omega\right) \\
\mathrm{m} \tilde{\xi}_{c g} \tilde{\Omega} V+\tilde{\Omega} J \Omega
\end{array}\right\} d s, \quad Q_{s t i f}=\int_{0}^{l} W^{T}\left\{\begin{array}{c}
\tilde{F} K-F^{\prime} \\
\tilde{M} K-M^{\prime}+\tilde{F}\left(e_{1}+\gamma\right)
\end{array}\right\} d s, \quad Q_{e x t}=\int_{0}^{l} W^{T}\left\{\begin{array}{l}
f \\
m
\end{array}\right\} d s,
$$

where $F$ and $M$ are the internal forces and moments defined in Eq. (2). Eqs. (24) are complemented by a problemdependent set of initial and boundary conditions. The system matrices $\mathcal{M}$ and $\mathcal{T}_{1}$ are constant (banded) matrices and only need to be inverted once and the problem can be easily posed as

$$
\begin{aligned}
& \dot{\mathbf{x}}=\mathbf{f}(\mathbf{x})+\mathbf{f}_{\text {ext }}, \\
& \mathbf{y}=\mathbf{g}(\mathbf{x}),
\end{aligned}
$$

where the state and output variables been defined, respectively, as 


$$
\mathbf{x}=\left\{\begin{array}{l}
\bar{V} \\
\bar{\Omega} \\
\bar{F} \\
\bar{M}
\end{array}\right\} \quad \text { and } \quad \mathbf{y}=\left\{\begin{array}{l}
\bar{R} \\
\bar{\Psi}
\end{array}\right\} .
$$

The functional $\mathbf{g}(\mathbf{x})$ is obtained from Eq. (12) if the internal forces and moments in each element are approximated by their value at the element midpoint. It is important to remark that, as velocities and strains are expressed in the material frame, the matrices $\mathcal{M}$ and $\mathcal{T}_{1}$ in Eq. (24) are constant matrices and they only need to be inverted once to pose the problem in the state-space form of Eq. (28). Note also that $\mathbf{f}(\mathbf{x})$ includes only quadratic nonlinearities, as the finite rotations only appear in the functional $\mathbf{g}(\mathbf{x})$ in the output equation and this will improve the convergence rate of the solution algorithms as compared to the displacement- or strain-based elements introduced above. This form of the equations is therefore particularly suitable for integration together with the $1^{\text {st }}$ order different equations that describe the rigid-body dynamics of the flexible aircraft in flight mechanics analysis.

As discussed before, this definition for an intrinsic element ( $i$-beam element) also only works for open kinematic chains on a single support. As with the strain-based element above, these equations would need to be complemented by additional constraints (through, for example, Lagrange multipliers) in the case of closed chains, and the reader is referred to Ref. 17 for more details on how this problem can be solved. The linearized expressions of Eqs. (24) can be easily obtained as

$$
\begin{gathered}
\mathcal{M} \frac{d}{d t}\left\{\begin{array}{l}
\Delta \bar{V} \\
\Delta \bar{\Omega}
\end{array}\right\}+C\left(V_{0}, \Omega_{0}\right)\left\{\begin{array}{l}
\Delta \bar{V} \\
\Delta \bar{\Omega}
\end{array}\right\}+\mathcal{D}\left(F_{0}, M_{0}\right)\left\{\begin{array}{l}
\Delta \bar{F} \\
\Delta \bar{M}
\end{array}\right\}=\Delta Q_{e x t}, \\
\mathcal{T}_{1} \frac{d}{d t}\left\{\begin{array}{l}
\Delta \bar{F} \\
\Delta \bar{M}
\end{array}\right\}+\mathcal{T}_{\gamma}\left(F_{0}, M_{0}\right)\left\{\begin{array}{l}
\Delta \bar{V} \\
\Delta \bar{\Omega}
\end{array}\right\}+\mathcal{T}_{v}\left(V_{0}, \Omega_{0}\right)\left\{\begin{array}{l}
\Delta \bar{F} \\
\Delta \bar{M}
\end{array}\right\}=0,
\end{gathered}
$$

where the new matrices are,

$$
\begin{gathered}
C=\int_{0}^{l} W^{T}\left[\begin{array}{cc}
\mathrm{m} \tilde{\Omega} & \mathrm{m}\left(\tilde{\xi}_{c g} \tilde{\Omega}-2 \tilde{\Omega} \tilde{\xi}_{c g}-\tilde{V}\right) \\
\mathrm{m} \tilde{\xi}_{c g} \tilde{\Omega} & \tilde{\Omega} J-\widetilde{J \Omega}-\mathrm{m} \tilde{\xi}_{c g} \tilde{V}
\end{array}\right] N d s, \\
\mathcal{D}=\int_{0}^{l} W^{T}\left[\begin{array}{cc}
0 & \tilde{F} \\
\tilde{F} & \tilde{M}
\end{array}\right]\left[\begin{array}{cc}
c_{\gamma f} & c_{\gamma m} \\
c_{\kappa f} & c_{\kappa m}
\end{array}\right] N d s-\int_{0}^{l} W^{T}\left[\begin{array}{cc}
\tilde{K} & 0 \\
\tilde{e}_{1}+\tilde{\gamma} & \tilde{K}
\end{array}\right] N d s-\int_{0}^{l} W^{T} N^{\prime} d s, \\
\mathcal{T}_{v}=\int_{0}^{l} W^{T}\left[\begin{array}{cc}
\tilde{\Omega} & \tilde{V} \\
0 & \tilde{\Omega}
\end{array}\right]\left[\begin{array}{cc}
c_{\gamma f} & c_{\gamma m} \\
c_{\kappa f} & c_{\kappa m}
\end{array}\right] N d s .
\end{gathered}
$$

Note that the linearized system matrices in Eq. (30) are not symmetric. For the particular case of non-gyroscopic reference conditions (i.e., $V_{0}=0, \Omega_{0}=0$ ), the natural frequencies in the linear problem defined by Eq. (30) are given by

$$
\left[\mathcal{D} \mathcal{T}_{1}^{-1} \mathcal{T}_{\gamma}+\omega^{2} \mathcal{M}\right]\left\{\begin{array}{l}
\phi_{V} \\
\phi_{\Omega}
\end{array}\right\}=0,
$$


where $\phi_{V}$ and $\phi_{\Omega}$ are the components of the mode shapes in the translational and angular velocities, respectively. The components of the mode shapes in the internal forces and moments at the nodes, $\phi_{F}$ and $\phi_{M}$, respectively are computed from

$$
\left\{\begin{array}{c}
\phi_{F} \\
\phi_{M}
\end{array}\right\}=\frac{i}{\omega} \mathcal{T}_{1}^{-1} \mathcal{T}_{\gamma}\left\{\begin{array}{c}
\phi_{V} \\
\phi_{\Omega}
\end{array}\right\} .
$$

As a result, the velocity contributions to the mode shapes are vectors in the real domain, while the strain contributions are imaginary numbers. Using the notation of Eq. (28), the linearized system of equations (30) can also be written in the usual linear state-space form, that is,

$$
\Delta \dot{\mathbf{x}}=\mathbf{A}\left(\mathbf{x}_{0}\right) \Delta \mathbf{x}+\Delta \mathbf{f}_{\text {ext }}
$$

\section{Model Substructuring}

\section{A. Combining displacement- and strain-based beam elements}

For open kinematic chains, strain-based solution methods (either $s$-beams or $i$-beams) become very efficient for geometrically-nonlinear problems, as they present quadratic convergence rates instead of the linear convergence achieve by displacement-based models. Only a few iterations are then needed to achieve convergence on complex load cases with large deformations. They are particularly fast in the case of follower loads, in which case there is no need to integrate the strains into displacements and rotations during the solution process. More on this is presented in the evaluation of numerical studies. The advantage of these methods is not so clear for more complex closed chains (for instance, a clamped-clamped arch or joined-wing aircraft ${ }^{10,17}$ ), although this would not be a concern for conventional wing-body-fuselage aircraft configurations. In situations with multiple interconnected bodies displacement-based elements could be more efficient.

It is however relatively simple to combine strain-based elements and displacement-based elements in a single model, so that the appropriate elements can be used in different parts of the vehicle. A typical example would be the use of linearized displacement-based beam elements for a fuselage and tail of a conventional aircraft configuration, with geometrically-nonlinear intrinsic beam elements for the high-aspect ratio wings. The dynamic equations of the combined system can be written as

$$
\begin{aligned}
& \mathcal{F}_{d}=0, \\
& \mathcal{F}_{d s}=F_{e x t, s}, \\
& Q_{s}=0,
\end{aligned}
$$

where $F_{d}$ are the discrete dynamic equations (6) at the internal nodes of the displacement-based elements, $Q_{s}$ are the discrete dynamic equations (24) on the intrinsic elements at internal nodes, and $F_{d s}$ are the nodal equations (6) at the interface nodes, on which the effect of the intrinsic elements is included by the instantaneous boundary force and 
moments at the attached end. These equations are complemented by imposing the same velocities and displacements at the interface nodes. Note that this implies only minor changes to the separate implementation of each formulation.

\section{B. Model reduction methods}

A further improvement in efficiency can be obtained if the part of the structure represented by linear elements is characterized by its (constrained or unconstrained) natural vibration modes. Several procedures are available in the literature and we follow the Craig-Bampton method ${ }^{15}$. In this procedure, the linear substructure (i.e., fuselage and tail) is first substituted by statically-reduced mass and stiffness matrices at the boundary nodes, which are added to the rest of the model. This is therefore the same as the Guyan coordinate elimination method, for which a transformation matrix is easily obtained from the response to unit variation on each degree of freedom at the boundary nodes. Additional states, not present in the Guyan reduction, are then added to model the dynamics of the linear substructure. They are obtained from the natural vibration modes of the linear substructure with constraints at the interface nodes.

In the current context, the displacement-based beam elements seem the most appropriate to model the linear beam dynamics, as they yield the standard second-order linear different equations with symmetric matrices. However, this approach could also be used if the information about the substructure to be reduced is obtained from any standard finite-element package. The integration with the intrinsic beam equations for the substructures with large displacements is better done by transforming the second-order differential equations on the linear modes into first-order state-space form.

\section{Numerical studies}

The different beam models described above have been implemented. Previous works ${ }^{10,14,17}$ by the second author and co-workers have extensively explored the details in the implementation of a strain-based model ( $s$-beam) and we will emphasize here more the $d$ - and $i$-beams. Results obtained with 2- and 3-noded displacement-based ( $d$-beam) elements and with a 2-noded intrinsic ( $i$-beam) element are presented, along with 2-noded constant strain ( $s$-beam) elements. The first observation from the numerical tests is that, in a Newton-Raphson-based solution procedure, the strain-based formulation shows quadratic convergence rates, as compared to the linear convergence of the displacement-based model. The main reason is that the linearization in strains still keeps the nonlinear straindisplacement relations after each iteration, while those relations are linearized on each iteration of the displacementbased element. Some test cases were defined to perform a partial validation of the implementation, including static and dynamic cases as follows.

\section{A. Static solutions}

The first test case corresponds to a cantilever 45 -degrees bend under a tip follower force ${ }^{15,20}$. The bend ${ }^{20}$ has a radius of $100 \mathrm{~m}$, a square cross section of side $1 \mathrm{~m}$, Young's modulus $\mathrm{E}=10^{7} \mathrm{~Pa}$ and negligible Poisson ratio. Figure 1 shows the tip displacements and the final deformed shape obtained with 8 intrinsic elements and compares 
the first with the results using a displacement-based model from Ref. 20, which required 8 load increments. The strain-based model obtains a solution without the need of applying the force in steps and with no more than 8 iterations for a result with a relative error smaller than $10^{-8}$.
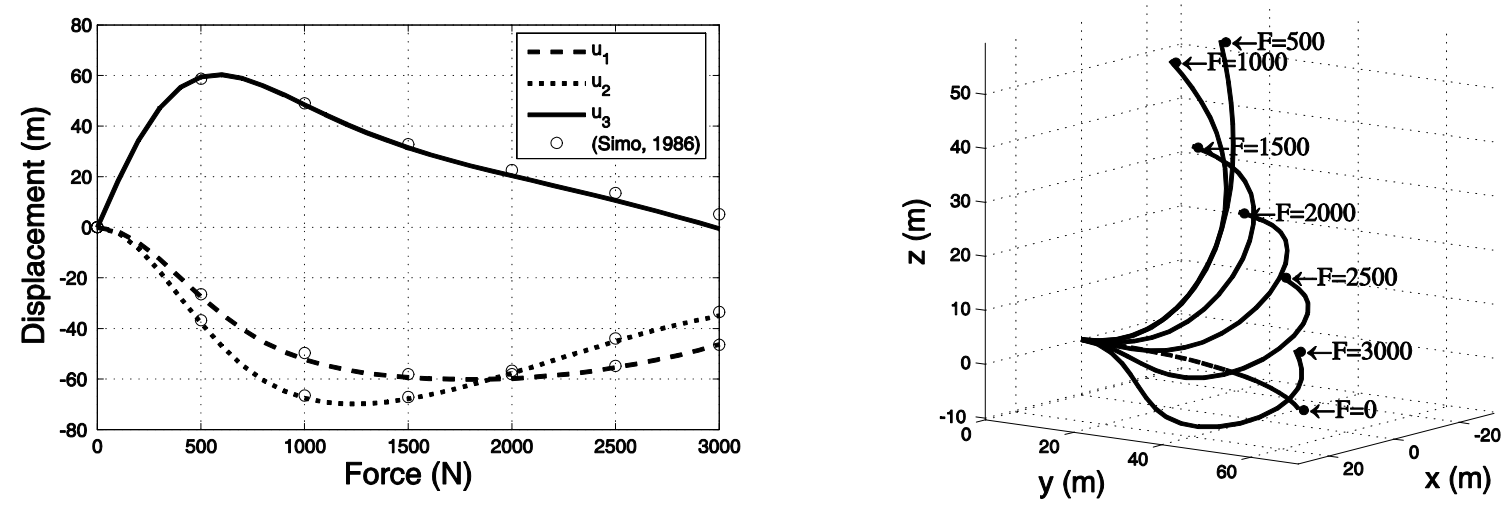

Figure 1. Components of the tip displacement (left) and deformed shape (right) for different applied follower tip loads for the $45^{\circ}$ cantilever bend. Present results based on $i$-beams.

For dead loads, the intrinsic model needs some more iterations as one now needs to compute at each step the global rotation at the point of application of the load. As an example, the 45-degrees bend described above was subject to a tip vertical load of $300 \mathrm{~N}$ and results are shown in Table 1. Both the displacement-based and the intrinsic model were used with 20-element 2-noded discretizations and they are compared with the results of the models introduced in Refs. 15 and 20. Results are in very good agreement. However, the displacement-based model required 22 load increments to converge and no less than 10 iterations on each increment, while the strain-based element converged in 36 iterations without any load substepping. It should also be pointed out that in the first implementation of the models, we have not yet consider the state-dependent contributions of the forcing term to the linearized equations on each increment and there is still room for even better performance of the intrinsic beam model in this case. An additional advantage of this model is that it is based on simpler algorithms that require around half the number of operations per iteration.

\begin{tabular}{lccc}
\hline \hline \multicolumn{4}{c}{ Table 1. Tip displacements for $\mathbf{4 5}^{\mathbf{0}}$ bend subject to a dead vertical force at the tip of $\mathbf{3 0 0} \mathbf{~ N}$} \\
\hline \hline & $\mathrm{U}_{1}(\mathrm{~m})$ & $\mathrm{U}_{2}(\mathrm{~m})$ & $\mathrm{U}_{3}(\mathrm{~m})$ \\
\hline Displacement-based element & 21.90 & 59.10 & 40.05 \\
Intrinsic element & 22.25 & 58.79 & 40.17 \\
Simo and Vu-Quoc, 1986 & 22.33 & 58.84 & 40.08 \\
Geradin and Cardona, 2001 & 22.14 & 58.64 & 40.35 \\
\hline \hline
\end{tabular}

As it was described above, the advantage of the intrinsic model to consider large deformations can be even more dramatic if we complement it with model substructuring techniques that split the problem in the regions of small and large displacements, typically, fuselage/tail and wings, respectively. Such techniques also allow for the direct use of modal information of the fuselage obtained from more complex finite-element models, to which geometricallynonlinear models of the wings can be attached. To investigate this, a simpler wing-body-tail beam model is 
proposed. Fuselage length is $12 \mathrm{~m}$ and wing span $30 \mathrm{~m}$. It has a T-tail 3-m high and 6-m span. Isotropic properties were defined throughout, and the relevant constants are: $\mathrm{EA}=5 \times 10^{8} \mathrm{~N}, \mathrm{kGA}=10^{8} \mathrm{~N}$, for extension and shear throughout the aircraft, respectively, bending stiffness equal to $\mathrm{EI}=5 \times 10^{6} \mathrm{~N} \cdot \mathrm{m}$ at the fuselage and vertical tail, $\mathrm{EI}=5 \times 10^{5} \mathrm{~N} \cdot \mathrm{m}$ in the horizontal tail, and $\mathrm{EI}=10^{6} \mathrm{~N} \cdot \mathrm{m}$ along the wings. Constant normal (initially vertical) followerforces are applied on the wings and horizontal tails and the model is clamped at the nose.

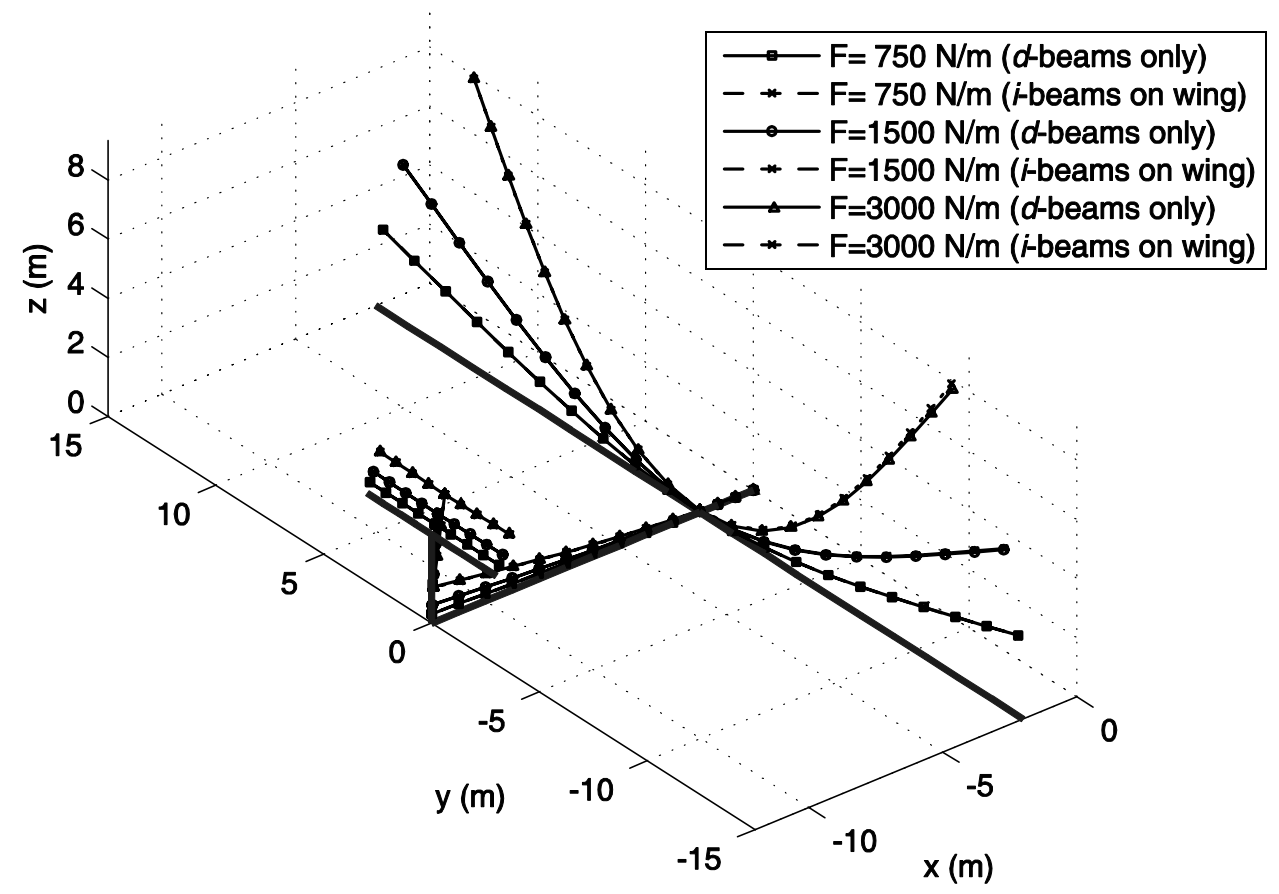

Figure 2. Deformation of wing-body-tail configuration for follower distributed load on wing and tail.

On this configuration, two models are studied. The first one is a standard model using displacement-based elements ( $d$-beams). A second model is obtained by using intrinsic elements only on the wings ( $i$-beams). The deformed configurations for different load levels are shown in Figure 2, and they show very good matching of the results obtained from both models. However, the model with intrinsic elements for the wing runs one order of magnitude faster than the model with only displacement-based elements. For a $3000 \mathrm{~N} / \mathrm{m}$ load level, the latter needs at least 23 load subiterations, while the former needs only 9 (larger than before because $d$-beams are still present in the model). In terms of computer time, for that case the use of $d$-beams only took 4.96 times more than when wings were modeled using the $i$-beams. Further improvement could be obtained if the elements in the fuselage and tail are linearized, but this has not been investigated here.

\section{B. Dynamic solutions}

The experimental results on very flexible Titanium beams obtained by $\mathrm{Pai}^{21}$ set the reference for this section. The dimensions of the beam are $479.0 \times 50.8 \times 0.45 \mathrm{~mm}$, with mass density $4,430 \mathrm{~kg} / \mathrm{m}^{3}$, Young's modulus $127 \mathrm{GPa}$, and Poisson's ratio 0.36 . The beam is highly flexible and a 40 -element $i$-beam model gives a $27.06 \%$ static tip deflection 
(normalized with respect to the beam length) when the beam is horizontal and subject to its own weight. Experiments in Ref. 21 were performed on an upward beam on a moving base with harmonic oscillations. The weight loads on the beam have to be included as they can have a significant contribution in the amplitude of the outof-plane motions. The first four experimental resonant frequencies -including the effect of the weight loads- $\operatorname{are}^{21}$ $1.41 \mathrm{~Hz}, 10.44 \mathrm{~Hz}, 29.72 \mathrm{~Hz}$, and $58.28 \mathrm{~Hz}$. Figure 3 shows the out-of-plane component of the velocity for two different excitations of the base. Results are normalized with the amplitude of the velocity at the base, $V_{\text {base }}$. Time step in all cases is chosen as $\Delta t=0.01 / f_{\text {base }}$, where $f_{\text {base }}$ is the frequency of the oscillation at the base.
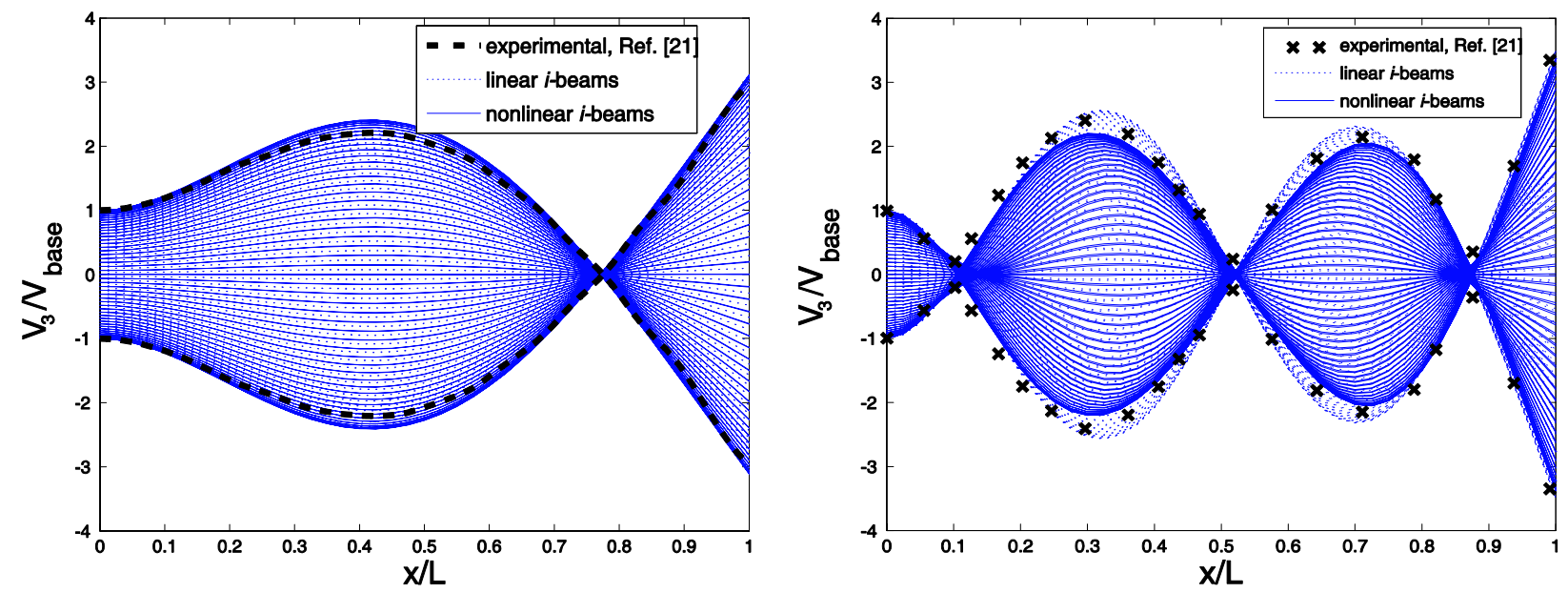

Figure 3. Normalized normal velocity profiles at 100 consecutive steps during one period of base oscillations (left: $V_{\text {base }}=0.1399 \mathrm{~m} / \mathrm{s}, f_{\text {base }}=9 \mathrm{~Hz}$; right: $V_{\text {base }}=0.3414 \mathrm{~m} / \mathrm{s}, f_{\text {base }}=32 \mathrm{~Hz}$ ). Coarse $i$-beam model (40 elements).

Numerical simulations in Figure 3 were carried out using the intrinsic beam model ( $i$-beam element) with linear spatial interpolation and an implicit mid-point rule for time-integration. It solves Eq. (24) for the nonlinear case and Eq. (30) for the linear one. The nonlinear solution is therefore equivalent to the finite-difference solution process proposed in Ref. 19 and results in an energy-preserving time integration scheme. Figure 3 compares the linear and nonlinear $i$-beam solutions using 40 beam elements with the envelope of the local velocities measured in Ref. 21 using a laser vibrometer. Results show very good agreement with the experimental results, in both the amplitudes of the beam motions and the location of the nodes. The fourth natural frequency is about twice the value of the third one and the beam shows a two-to-one internal resonance when excited at $32 \mathrm{~Hz}$. As a result, even though beam displacements are very small (with a maximum of about $2 \%$ of the beam span), there are differences between the linear and the nonlinear model due to the energy transfer between modes in the (nonlinear) internal resonance ${ }^{22}$. This is verified by the experimental results in Ref. 21, which show the same shift on the maximum and do not show clear nodal points. Further refinement of the model shows more clearly the effect of internal resonance, as can be seen in Figure 4 for results obtained with 40 and $60 i$-beam elements. As there is no structural damping in the model, however, the high-frequency contributions are magnified in the numerical results with respect to those obtained in the experiments. Contributions at the frequencies $3^{\text {rd }}$ and $4^{\text {th }}$ vibration modes can be easily observed on the timeevolution of the vertical tip velocity in Figure 4 when the model is refined. 

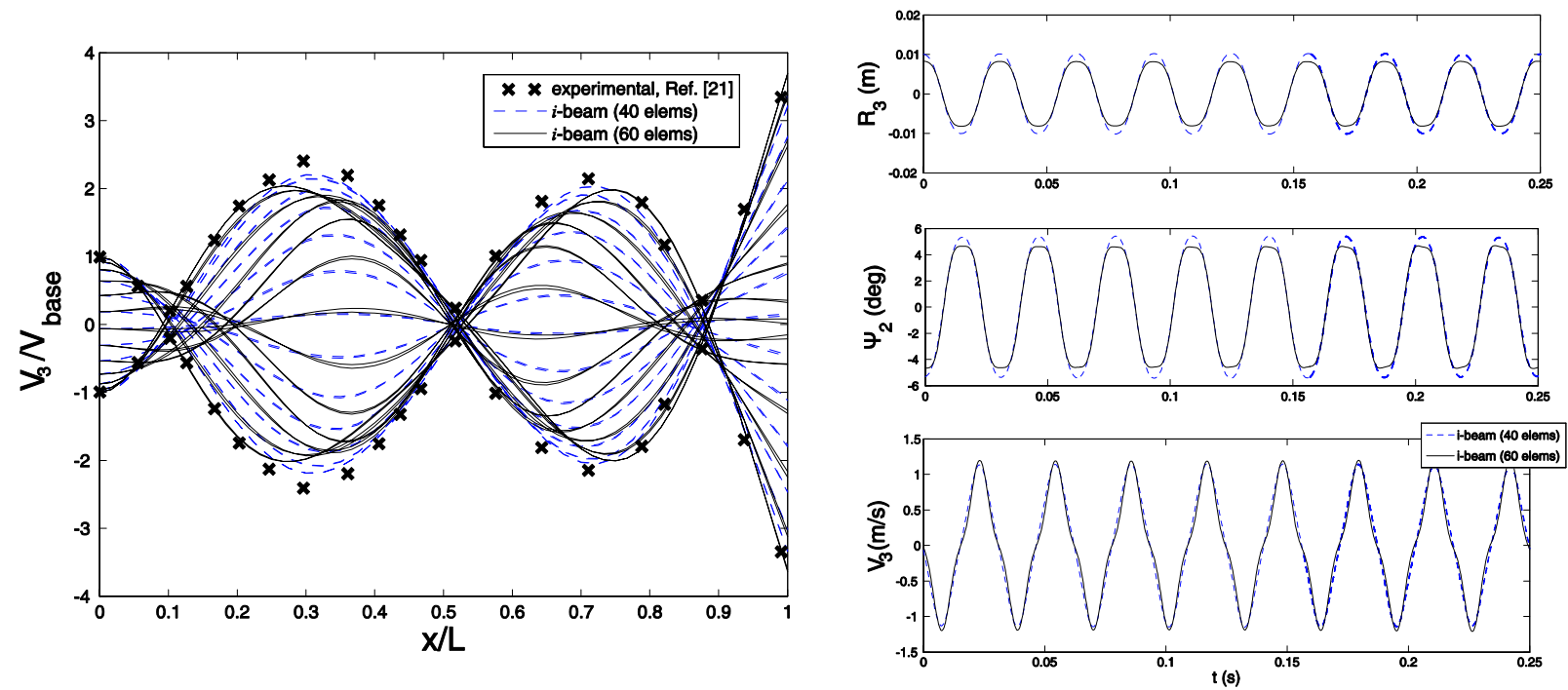

Figure 4. Effect of model refinement on beam with oscillating base $\left(V_{\text {base }}=0.3414 \mathrm{~m} / \mathrm{s}, f_{\text {base }}=32 \mathrm{~Hz}\right)$. Left: Normalized normal velocity profiles at 25 steps during one period. Right: tip displacement, bending rotation and velocity. Nonlinear intrinsic beam models.

Figure 5 shows the results obtained with the implementation of strain-based beam elements ( $s$-beams) of Ref. 17. In this case the contribution of the shear strains has been neglected and the discretization is such that the dynamic equations are satisfied at each node instead of at the element centers. As a result, a discretization with 40 elements has enough accuracy to capture the nonlinear interaction between the third and fourth modes for the $32-\mathrm{Hz}$ excitation at the base. Results using $s$-beams in Figure 5 match very well those obtained by $i$-beams in Figure 4 for high the frequency excitations and those of Figure 3 for the $9-\mathrm{Hz}$ case. There is however a larger number of operations in each iteration of the s-beam model, and computational time in this case was almost an order of magnitude larger than for the model based on $i$-beams. However, the implementation was done in a completely different setup (including the use of Matlab instead of Fortran) and this comparison, while showing the expected trend, might not accurately reflect the actual different performance of both models.
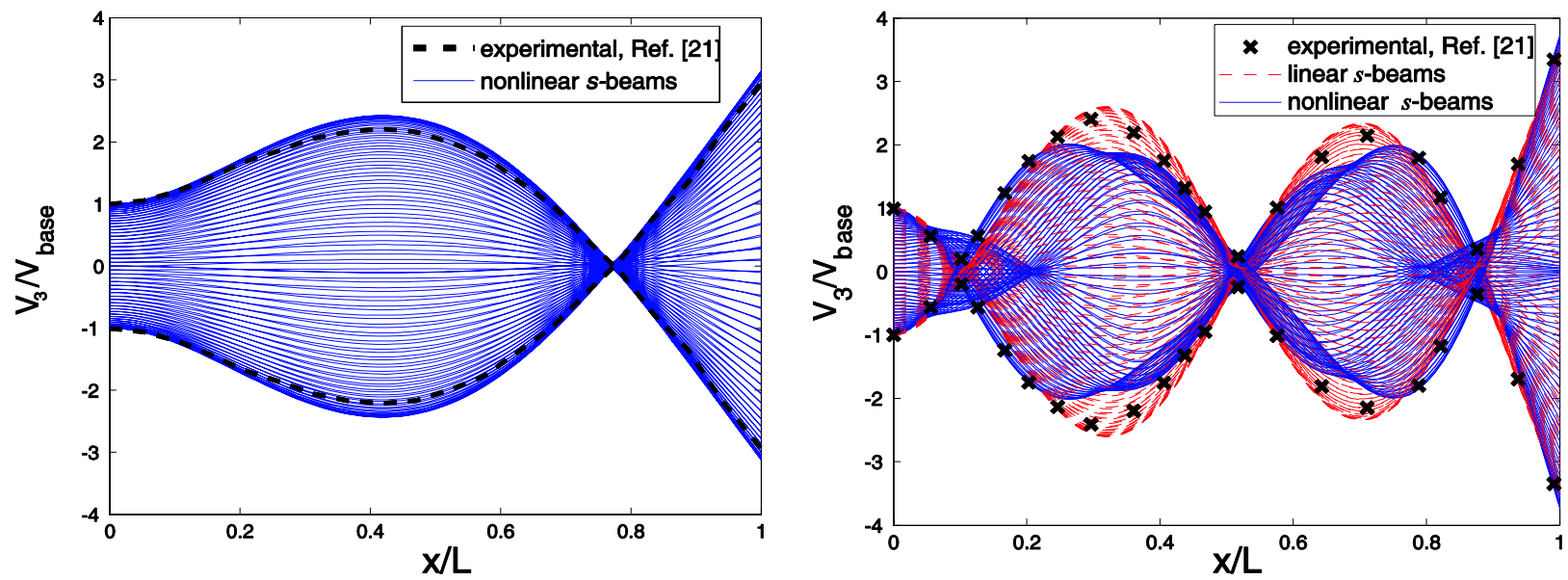

Figure 5. Normalized normal velocity profiles at 100 consecutive steps during one period of base oscillations (left: $V_{\text {base }}=0.1399 \mathrm{~m} / \mathrm{s}, f_{\text {base }}=9 \mathrm{~Hz}$; right: $V_{\text {base }}=0.3414 \mathrm{~m} / \mathrm{s}, \mathrm{f}_{\text {base }}=32 \mathrm{~Hz}$ ). Strain-based beam model. 


\section{Conclusions}

The solution to the geometrically-nonlinear beam equations can be obtained with different set of independent degrees of freedom. We have investigated three of them in the context of the structural modeling required for coupled aeroelastic/flight dynamic analysis of very flexible aircraft. It has been found that the computational cost of conventional displacement-based models can be significantly reduced by using a description based on the intrinsic beam equations, in which velocities and strains define the independent structural states of the vehicle. A strain-based model, based on beam strains and their time derivatives offers another promising approach to the same problem. As with the intrinsic model, it does not need to integrate finite rotations, and in this case it also preserves the symmetry of the system (and the second-order-differential-equation form). We have also extended the description of the strainbased model that we had used in previous works to include the shear strains as independent degrees of freedom. Results have shown the effectiveness of those alternative beam models to address problems with geometricallynonlinear effects. We have exemplified this with static problems with large displacements and dynamic problems with internal resonances. Linear structural results in those cases need to be refined and we have shown the efficiency of strain-based approaches to provide the nonlinear description. Furthermore, a (nonlinear) state-space form of the discrete intrinsic beam equations provides a natural description of the vehicle structural dynamics for integration in flight mechanic analysis.

The ability to combine the different elements may result however in the most efficient solution. In particular, we have investigated the use of displacement-based elements for those parts of the structure which are subject to small deformations (i.e., fuselage and tail), while intrinsic or strain-based elements are used to model the flexible wings. Further refinements are possible if modal reduction techniques (in particular, the Craig-Bampton method) are used on the linear substructures.

\section{Acknowledgments}

The authors are grateful to Weihua Su (University of Michigan) for conducting the numerical simulations using the strain-based beam models.

\section{References}

${ }^{1}$ Rodden WP, Love JP (1985). "Equations of Motion of a Quasisteady Flight Vehicle Utilizing Restrained Static Aeroelastic Characteristics." Journal of Aircraft 22 (9) pp. 802-809.

${ }^{2}$ Meirovitch L, Tuzcu I (2004). "Unified Theory for the Dynamics and Control of Maneuvering Flexible Aircraft.” AIAA Journal 42 (4) pp. 714-727.

${ }^{3}$ Baldelli DH, Chen PC, Panza J (2006). "Unified Aeroelastic and Flight Dynamic Formulation via Rational Function Approximations." Journal of Aircraft 43 (3) pp. 763-772.

${ }^{4}$ Noll TE, Brown JM, Perez-Davis ME, Ishmael SD, Tiffany GC, Gaier M (2004). Investigation of the Helios Prototype Aircraft Mishap. NASA Technical report [http://www.nasa.gov/pdf/64317main helios.pdf, accessed 01.04.2009].

${ }^{5}$ Van Schoor MC, von Flotow AH (1990). “Aeroelastic Characteristics of a Highly Flexible Aircraft.” Journal of Aircraft 27 (10) pp. 901-908. 
${ }^{6}$ Drela M (1999). "Integrated Simulation Model for Preliminary Aerodynamic, Structural, and Control-Law Design of Aircraft.” AIAA Paper No. 1999-1394.

${ }^{7}$ Patil MJ, Hodges DH, Cesnik CES (2000). "Nonlinear Aeroelastic Analysis of Complete Aircraft in Subsonic Flow." Journal of Aircraft 37 (5), pp. 753-760.

${ }^{8}$ Shearer CM, Cesnik CES (2007). "Nonlinear Flight Dynamics of Very Flexible Aircraft." Journal of Aircraft 44 (5) pp. 1528-1545.

${ }^{9}$ Palacios R, Cesnik CES, Reichenbach EY (2007). "Re-examined Structural Design Procedures for Very Flexible Aircraft." 2007 International Forum of Aeroelasticity and Structural Dynamics, Stockholm, Sweden.

${ }^{10}$ Cesnik, CES, Brown, EL (2002). "Modeling of High Aspect Ratio Active Flexible Wings for Roll Control." AIAA Paper No. 2002-1719.

${ }^{11}$ Palacios R, Cesnik CES (2005). "Cross-Sectional Analysis of Non-Homogeneous Anisotropic Active Slender Structures.” AIAA Journal 43 (12) pp. 2624-2638.

${ }^{12}$ Peters DA, Karunamoorthy S, Cao W (1995). "Finite State Induced Flow Models. Part I: Two-Dimensional Thin Airfoil." Journal of Aircraft 32 (2) pp. 313-322.

${ }^{13}$ Laxman V, Venkatesan C (2007). "Chaotic Response of an Airfoil due to Aeroelastic Coupling and Dynamic Stall.” AIAA Journal 45 (1) pp. 271-280.

${ }^{14}$ Shearer CM, Cesnik CES (2006). "Modified Generalized- $\alpha$ Method for Integrating Governing Equations of Very Flexible Aircraft.” AIAA Paper No. 2006-1747.

${ }^{15}$ Géradin M, Cardona A (2001). Flexible Multibody Dynamics. A Finite Element Approach. John Wiley and Sons, Ltd, Chichester, England.

${ }^{16}$ Hodges DH (1990). "A Mixed Variational Formulation Based on Exact Intrinsic Equations for Dynamics of Moving Beams." International Journal of Solids and Structures 26 (11) pp. 1253-1273.

${ }^{17}$ Cesnik CES, Su W (2005). "Nonlinear Aeroelastic Modeling and Analysis of Fully Flexible Aircraft." AIAA Paper No. 2005-2169.

${ }^{18}$ Palacios R, Cesnik CES (2008). "On the One-dimensional Modeling of Camber Bending Deformations in Active Aanisotropic Slender Structures." International Journal of Solids and Structures 45 (7-8) pp. 2097-2116.

${ }^{19}$ Hodges DH (2003). "Geometrically Exact, Intrinsic Theory for Dynamics of Curved and Twisted Anisotropic Beams.” AIAA Journal 41 (6) pp. 1131-1137.

${ }^{20}$ Simo JC, Vu-Quoc L (1986). "A Three-Dimensional Finite- Part II: Computational Aspects." Computer Methods in Applied Mechanics and Engineering 58, pp. 79-116.

${ }^{21}$ Pai PF (2007). Highly Flexible Structures: Modeling, Computation, and Experimentation. AIAA Education Series. AIAA, Reston, Virginia, USA.

${ }^{22}$ Nayfeh AH (2000). Nonlinear Interactions: Analytical, Computational, and Experimental Methods. John Wiley \& Sons, Chichester, New York, USA. 